\title{
Evidence for Muscle-Dependent Neuromuscular Synaptic Site Determination in Mammals
}

\author{
Vita M. Vock, ${ }^{1}$ Olga N. Ponomareva, ${ }^{1}$ and Mendell Rimer ${ }^{1,2}$ \\ ${ }^{1}$ Section of Neurobiology, University of Texas at Austin, Austin, Texas 78712, and ${ }^{2}$ Department of Neuroscience and Experimental Therapeutics, College of \\ Medicine, Texas A\&M Health Science Center, College Station, Texas 77843
}

\begin{abstract}
Recent evidence challenges the prevalent view that neural factors induce the formation of a de novo postsynaptic apparatus during development of the vertebrate neuromuscular junction. The latest experiments suggest an alternative model in which the muscle fiber induces a nascent postsynaptic apparatus and sets the location of the future synapse. On axonal contact, these sites, laid out in a prepattern in the central area of developing muscle fibers, mature into synapses by the combined action of neural factors such as agrin and ACh. We sought to test in mammals these two models of neuromuscular synaptogenesis. Previously, we showed that continuous prenatal muscle expression of constitutively active ErbB2 (CAErbB2) led to synaptic loss, exuberant axonal sprouting, and lethality at birth. Here, we transiently induced CAErbB2 during midgestation and examined synapse restoration after inducer withdrawal. Centrally enriched $A C h$ receptor $(A C h R)$ transcription and clustering were abolished after transient CAErbB2 induction. After inducer withdrawal, synapses were restored but were distributed widely over the entire diaphragm muscle. Under the nerve-dependent model, this distribution is explained by the wide pattern of axonal sprouting triggered by CAErbB2. Yet, in the absence of the nerve, introduced in our animals by mating to $\mathrm{Hb}^{+1-}$ mice, a very similar, wide distribution of aneural AChR clusters resulted. Thus, transient expression of CAErbB2 in skeletal muscles leads to reprogramming of the endogenous muscle AChR prepattern. This, and not the nerve, seems primarily responsible for the widely distributed pattern of synapses in our experimental animals.
\end{abstract}

Key words: acetylcholine receptor; motoneuron; muscle fiber; agrin; neuromuscular junction; synaptogenesis

\section{Introduction}

The vertebrate neuromuscular junction (NMJ), the synapse between a motor neuron and a skeletal muscle fiber, is the best studied of all synapses, and it continues to provide important insights into general synaptogenesis throughout the nervous system. In the now classic paradigm, axons contact developing muscle fibers at random sites and release agrin, a proteoglycan that induces acetylcholine receptor (AChR) clustering, and other aspects of postsynaptic differentiation, on activation of a muscle membrane receptor complex containing the muscle-specific receptor tyrosine kinase MuSK. Presynaptic differentiation follows, driven by retrograde signals from the muscle that transform the axonal growth cone into a nerve terminal. In this model, the nerve is the inducer of postsynaptic differentiation (McMahan, 1990). Neuromuscular junctions localize to an "endplate zone" in the middle of muscle fibers. According to the classic view, this

\footnotetext{
Received Nov. 15, 2007; revised Feb. 6, 2008; accepted Feb. 7, 2008.

This work was supported by National Institutes of Health Grant GM065797 and by setup funds from Texas A\&M Health Science Center (M.R.). We thank W. Thompson for stimulating discussions throughout. We are grateful to $L$. Chodosh, T. Jessell, S. Burden, and W. Thompson for kindly providing the breeding pairs for tet0-CAErbB2 ${ }^{+1-}$ $\mathrm{Hb}^{+/-}$, and S100-GFP mice, respectively. We also thank S. Burden for the plasmid probes for in situ hybridization and M. Rüegg for antiserum to MuSK.

Correspondence should be addressed to Mendell Rimer, Department of Neuroscience and Experimental Therapeutics, College of Medicine, Texas A\&M Health Science Center, 228 Reynolds Building, College Station, TX 778431114. E-mail: mjrimer@medicine.tamhsc.edu.

V. M. Vock's present address: Department of Cell Biology, University of Virginia, Charlottesville, VA 22908.

DOI:10.1523/JNEUROSCI.5080-07.2008

Copyright $\odot 2008$ Society for Neuroscience $\quad$ 0270-6474/08/283123-08\$15.00/0
}

location results from the initial contact by the nerve of short embryonic myotubes that later grow by fusion of more cells at their ends. Recently, however, this view has been challenged by evidence that suggest that the initial nerve-muscle contact is not random but is directed to the middle of the muscle fiber by a prepattern of AChR expression and clustering present in the central area of the fibers (Yang et al., 2000, 2001; Lin et al., 2001, 2005; Misgeld et al., 2005). Thus, NMJs localize to the endplate zone from the beginning of synaptogenesis as wandering axons preferentially contact preformed, nerve-independent AChR clusters in the central area. These sites mature into synapses by the action of nerve-derived synaptogenic signals such as agrin and acetylcholine. In this model, the muscle is the inducer of postsynaptic differentiation (for review, see Arber et al., 2002; Kummer et al., 2006).

The optical transparency of zebrafish embryos has made possible the testing of these models by vitally imaging whether the aneural AChR clusters are incorporated into nascent synapses. Results from these experiments suggest that these clusters are commonly (Panzer et al., 2006), but not exclusively (FlanaganSteet et al., 2005), the site of synapse formation. However, zebrafish and mammalian neuromuscular synaptogenesis differ in several important aspects (Panzer et al., 2005); thus, it remains open whether these results also apply to mammals. Live imaging of early mammalian synaptogenesis is currently unfeasible; nevertheless, another approach to examine the role of the prepattern is to probe whether embryonic axons can induce synapses de novo 
if they are driven to grow outside the central area of the muscle in which the prepattern localizes. In their simplest forms, the nervedependent model predicts that axons will induce synapses anywhere on the muscle fiber if given the chance, whereas the muscle-dependent (i.e., prepattern) model predicts they will not.

We recently showed (Ponomareva et al., 2006) that tetracycline-controlled, muscle-specific expression of a constitutively active ErbB2 (CAErbB2) receptor during embryogenesis produces a phenotype in which AChR clusters disperse by embryonic day 15.5 (E15.5), axons sprout along muscle fibers without stop, and synapses fail to form, leading to lethality at birth. As we also control the timing of CAErbB2 expression in these mice, here we asked whether and where synapses would form by birth after withdrawal of the antibiotic inducer. We find that axons continue to sprout after doxycycline (Dox) removal at E15.5 but that they seemingly stop at random to engage in synaptogenesis all along the entire width of the diaphragm muscle. Although, at first glance, this finding is consistent with the nerve-dependent model, we find that the same widely diffused pattern of AChR clusters is generated in diaphragms with similar transient expression of CAErbB2 but that lack altogether the phrenic nerve by genetic ablation of the Hb9 gene (Arber et al., 1999; Thaler et al., 1999). Thus, transient, skeletal muscle-specific expression of CAErbB2 leads to reprogramming of the endogenous muscle AChR prepattern. This, and not the nerve, seems primarily responsible for the widely distributed pattern of synapses in our experimental animals. These results appear to support a role for the muscle fiber in determining the site of initiation in mammalian neuromuscular synaptogenesis in vivo.

\section{Materials and Methods}

Mice. The transgenic lines harboring cDNAs encoding a reverse tetracycline transactivator (rtTA) or CAErbrB2 were described previously (Ponomareva et al., 2006). Homozygous CAErbB2 males were crossed to heterozygous rtTA females. Both single transgenic (ST) $\left(r t \mathrm{TA}^{-/-}\right.$; CAE$\left.r b B 2^{+/-}\right)$and bitransgenic (BT) $\left(r t \mathrm{TA}^{+/-}\right.$; CAErbB2 $\left.{ }^{+/-}\right)$embryos were generated from the same cross. The former (ST) served as control animals, whereas the latter (BT) were the experimental animals. Heterozygous $\mathrm{Hb} 9$ mice [kindly provided by S. Burden (New York University, New York, NY), with permission from T. Jessell (Columbia University, New York, NY)] were crossed into BT animals to generate BT progeny that was also HB9 null (BT $\mathrm{Hb}^{-/-}$) and lacked phrenic innervation of diaphragm as described previously (Arber et al., 1999). A transgenic line expressing green fluorescence protein (GFP) under the control of $S 100$ regulatory sequences [kindly provided by W. Thompson, University of Texas at Austin, Austin, TX (Zuo et al., 2004)] was crossed with the rtTA mice to produce females that were $G F P^{+/-} ; r t T A^{+/-}$. Crossing these females with homozygous CAErbB2 males produced ST and BT progeny that had GFP-labeled Schwann cells.

Mouse genotyping. Genomic DNA was prepared following standard methods. Genotyping was done by PCR using HotStart TaqDNA polymerase (Qiagen, San Diego, CA). Primers were as follows: (1) rtTA: forward, 5'-AGAGCACAGCGGAATGACTT-3'; reverse, 5'-GCCTGACGACAAGGAAACTC-3'; (2) CAErbB2:IRES:Luciferase: forward, 5'-CTTCTTCGCCAAAAGCACTC-3'; reverse, $5^{\prime}$-CACACAGTTCGCCTCTTTGA-3'; (3) HB9 deletion: forward, 5'-TGCACAGGCGGCTCTCTATGG-3'; reverse, 5'-CCACAGCTCGCTAGGAGGTGAG3'; (4) GFP: forward, 5'-AATCGAGTTGAAGGGCATTG-3'; reverse, 5'-GCCGATTGGAGTGTTCTGTT-3'.

Doxycycline induction. Dox (Sigma, St. Louis, MO) was mixed with customary ground mouse food, sucrose, and water. For $1 \mathrm{~g}$ of food, we added $6 \mathrm{mg}$ of Dox, $5 \%$ sucrose, and water to make a paste that was cut into pellets. Dox-containing food was given to pregnant dams at the time indicated in the text. Dox reached the embryos through the placenta. All animal experimentation was approved by the University of Texas Institutional Animal Care and Use Committee.
Luciferase assay. Muscles were homogenized in Luciferase Cell Culture Lysis Reagent (Promega, Madison, WI) and centrifuged at $4^{\circ} \mathrm{C}$. Twenty microliters of cleared lysate were added to a Chromalux flat-bottom plate (Dynex, Chantilly, VA). Luciferase activity assay was performed on a Dynex MLX Microplate Luminometer using $40 \mu \mathrm{l}$ of Luciferase Assay Reagent (Promega) with a total read time of $10 \mathrm{~s}$. Total light units obtained from reading were normalized to total protein content determined by Bradford assay (Bio-Rad, Hercules, CA).

Immunohistochemistry. Diaphragm muscles were immunostained as described previously (Ponomareva et al., 2006). E14 and E15 whole embryos were removed and fixed in $4 \%$ paraformaldehyde (PFA) at $4{ }^{\circ} \mathrm{C}$ overnight. The next day, concentrated PFA was replaced with $0.4 \%$ PFA until diaphragm dissection (1-4 d after embryo removal). Older (E16E18) embryos and neonates were fixed in 2\% PFA and replaced with $0.2 \%$ PFA as described above. Diaphragms were dissected and washed two times in PBS for 5 min each in a multiwell culture dish. Axons and nerve terminals were labeled with polyclonal rabbit anti-neurofilament (NF) at 1:500 (Millipore Bioscience Research Products, Temecula, CA) and anti-synaptophysin (syn) at 1:200 (Zymed, now Invitrogen, Carlsbad, CA). Rhodamine-conjugated, anti-rabbit IgG secondary (Jackson ImmunoResearch, West Grove, PA) was used to visualize neuronal markers, whereas AChRs were labeled with fluorescein- $\alpha$-bungarotoxin (BTX) (Invitrogen) at 1:1000. Samples were mounted in Vectashield (Vector Laboratories, Burlingame, CA) and viewed in a Eclipse E-1000 fluorescence microscope (Nikon, Tokyo, Japan) equipped with filters selective for either fluorescein or rhodamine.

In situ hybridization. Diaphragms from E15.5 embryos and postnatal day 0 (P0) were dissected and fixed overnight in 4\% PFA in PBS. A described previously, probe for $A C h R \alpha$ (Rimer et al., 2004) and a $1317 \mathrm{bp}$ probe for mouse $A C h R \varepsilon$ cDNA ( $A C h R \varepsilon$ ISH2; kindly provided by $\mathrm{S}$. Burden) were labeled with digoxigenin, chemically hydrolyzed (Schaeren-Wiemers and Gerfin-Moser, 1993), and used for wholemount in situ hybridization following a protocol originally described for tissue sections (Braissant and Wahli, 1998).

Confocal microscopy. High-resolution, high-magnification images were captured using a Leica (Nussloch, Germany) SP2 AOBS Laser Scanning Spectral Confocal Microscope. A $63 \times$ oil [HCX PL APO 1.4-0.60 numerical aperture (NA) Blau CS] objective with digital zoom allowed captured images at $\sim 120 \times$ magnification.

Regions of diaphragm muscle containing groupings of several synapses were first localized using a low-power objective lens. High-power images were obtained in which at least one cluster was visible en face, oriented toward the viewer. $Z$-stacks were $0.12 \mu \mathrm{m}$ thick. To measure postsynaptic area, stacks were rotated using the MetaMorph software tool (Molecular Devices, Downingtown, PA) until one cluster of the region was visible en face. A maximal projection was generated from the stack, and the border around the en face AChR cluster was thresholded. This was repeated for six en face clusters from each ST and BT animal. Area occupied by labeled AChRs was measured and Student's $t$ test was done to determine whether cluster area differed between control and experimental animals.

Aneural AChR cluster analysis. Diaphragm muscle from E16.5, E17.5, and $\mathrm{P} 0$ was visually scanned using the $40 \times$ objective (NA, 1.30; Nikon) in the wide-field microscope described above. Three to five regions of lateral, normally extrajunctional zone were examined in BT samples, whereas one to two regions adjacent to the center of the muscle were examined in ST samples. In all cases, regions selected were either dorsal or ventral to region of phrenic nerve branching. Clusters in a given field were counted and categorized as either nerve-associated (colocalized with nerve terminal staining) or aneural (not near a visible nerve terminal). Planes above and below the plane of focus for the AChR clusters were scanned to confirm the absence of nearby axonal processes.

Synapse/aneural AChR cluster pattern distribution analysis. Montages were created by aligning adjacent images of diaphragm muscle taken with the $10 \times$ objective (NA, 0.30; Nikon) of the wide-field microscope. All images were obtained from the region of diaphragm just ventral to the area in which phrenic nerve branching occurs. The width of hemidiaphragm was then determined by drawing a line across the width of the assembled collage. The midline of hemidiaphragm width was marked on 
each collage along with a short line, perpendicular to the width line and extending above the width line for a distance of $1 / 30$ of muscle width. The distance between random AChR clusters and the midline was measured and recorded. Sixty cluster distances were obtained from hemidiaphragms of three embryos for each genotype. ANOVA was calculated using distance measurements normalized to the total measured width of hemidiaphragm from each animal.

\section{Results}

\section{Removal of AChR clusters by E15.5}

Our laboratory showed previously that in vivo induction of a ligand-independent, constitutively active form of the ErbB2 receptor (CAErbB2), throughout murine embryogenesis resulted in the disappearance of AChR clusters from skeletal muscle (Ponomareva et al., 2006). To determine the extent of AChR cluster loss after a limited induction of the CAErB2 receptor, we used the previously described tet-on genetic system, which uses a CAErbB2 sequence downstream of a tetracycline inducible sequence. Heterozygous females for $r t \mathrm{TA}^{+/-}$were crossed with males homozygous for the CAErbB2 receptor $\left(C A E r b B 2^{+/+}\right)$to produce experimental and control animals in each litter. After verification of conception, expectant females were given doxycycline-containing rodent food for a limited period during gestation, beginning induction on E11.5 and continuing until E15.5 when Dox was removed from food. Litters were taken at E15.5, and whole-mount diaphragm immunostaining was performed to determine the extent of receptor cluster loss using epifluorescence microscopy. Axons and nerve terminals were visualized by using antibodies to NF and syn, whereas AChRs were labeled using fluorophore-conjugated BTX. During normal murine development, the phrenic nerve descends to the rostral side of diaphragm muscle in which thick fiber bundles extend and distribute throughout fibers. The bundles branch from a medial position to reach a central region of muscle fiber length and then extend in both ventral and dorsal directions, running perpendicular to the length of the myofibers. In ST animals $\left(\mathrm{rtTA}^{-/-} ; \mathrm{CAE}\right.$ $r b B 2^{+/-}$) AChR clusters were found colocalized with nerve terminals near a central region of muscle and proximal to the central nerve bundle (Fig. 1 A,B). After 4 d of CAErbB2 expression, however, diaphragm staining from BT animals $\left(r t \mathrm{TA}^{+/-}\right.$; $\mathrm{CAE}-$ $r b B 2^{+/-}$) showed a complete elimination of AChR clusters from the surface of the muscle (Fig. $1 C, D$ ). Thus, whether started at conception (i.e., E0.5) as in the study by Ponomareva et al. (2006) or at midgestation as done here, induction of CAErbB2 leads to AChR cluster loss by E15.5 in diaphragm muscle.

\section{Synapses form by birth throughout the entire width of diaphragm after inducer withdrawal}

We determined previously that, in addition to receptor loss, another consequence of CAErbB2 activation throughout gestation was extensive axonal outgrowth. Animals that had induced CAErbB2 signaling throughout gestation showed axons that extended from the central region of the diaphragm muscle to reach the distal regions of muscle fibers at E18.5 (Ponomareva et al., 2006). As a result of both AChR cluster elimination and nerve outgrowth, synapses failed to form in muscles expressing CAErbB2 (Ponomareva et al., 2006). We sought to restore synapse formation by withdrawing Dox-containing food at E15.5 and allowing elimination of CAErbB2 signaling for a period before birth. We reasoned that, because of the axonal outgrowth, synapses would have to form outside the central region of the muscle, in the absence of a preexisting AChR prepattern. Hence, this manipulation would allow us to test the two models of synapse initiation.
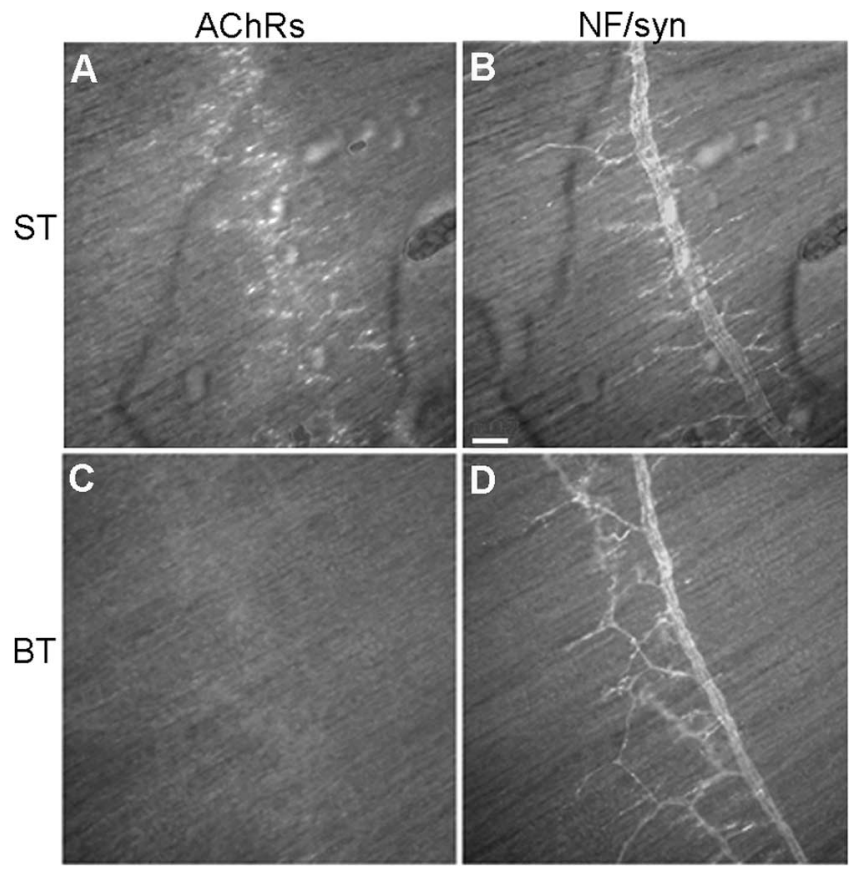

Figure 1. Disappearance of $A C h R$ clusters after transient CAErbB2 induction. Pregnant $r t T A^{+/-}$females, fertilized by $C A E r b B 2^{+/+}$males, were fed Dox-containing rodent food from E11.5 to E15.5. Whole mounts of diaphragm muscle from E15.5 ST $(\boldsymbol{A}, \boldsymbol{B})$ or BT $(\boldsymbol{C}, \boldsymbol{D})$ embryos were stained with rhodamine- $\alpha$-BTX and rabbit anti-NF and anti-syn antibodies. ST diaphragm shows a central location of both $A C h R s(\boldsymbol{A})$ and axons and nerve terminals $(\boldsymbol{B})$, whereas BT diaphragm shows the absence of $A C h R$ clusters $(\boldsymbol{C})$ in the area in which axons and nerve terminals are labeled. Scale bar, $50 \mu \mathrm{m}$.

The nerve-dependent model would predict that the nerve would be able to induce synapses anywhere along the surface of the muscle fiber, whereas the muscle-dependent model would predict that synapses would form only at sites in which there is a preexistent aneural AChR cluster. Bitransgenic embryos fed Dox throughout development (or starting at midgestation; data not shown) are stillborn because NMJs fail to form by birth. Hence, we anticipated that, if synapses were to form after Dox withdrawal but before delivery, BT pups would be able to survive at birth. Live BT $\left(\mathrm{CAErbB2} 2^{+/-} ; r t \mathrm{TA}^{+/-}\right)$mice were indeed recovered among pups at P0 in litters in which Dox was withdrawn at E15.5. Their frequency was consistent with a Mendelian distribution (14 litters, 127 total live pups, 66 ST, $61 \mathrm{BT}$ ). As expected, the survival of BT animals correlated with a drop in transgenic expression. Figure 2 shows that there was a 100 -fold decrease in transgene expression in BT embryos at E17.5 after Dox withdrawal at E15.5. No BT surviving pups were observed if Dox was withdrawn at E16.5 or later (data not shown). We performed immunostaining of whole-mount diaphragms from P0 pups to determine the location of synapses. Diaphragm muscle from survival animals was labeled for presynaptic and postsynaptic markers as above. Single-transgenic, control animals showed the expected central colocalization of nerve and receptor clusters (Fig. 3). Conversely, muscle staining from recovered BT neonates showed that synapses were not exclusively localized in the central zone of the muscle but that nerves had extended and grown out throughout the diaphragm as observed previously with CAErbB2 induction throughout gestation. Some nerve terminals and AChR clusters were seen colocalized even near the edges of diaphragm muscle (Fig. 3). High-power, confocal views of these synapses showed normal apposition between nerve terminals and AChRs (Fig. 4). No aneural AChR clusters were observed at P0 


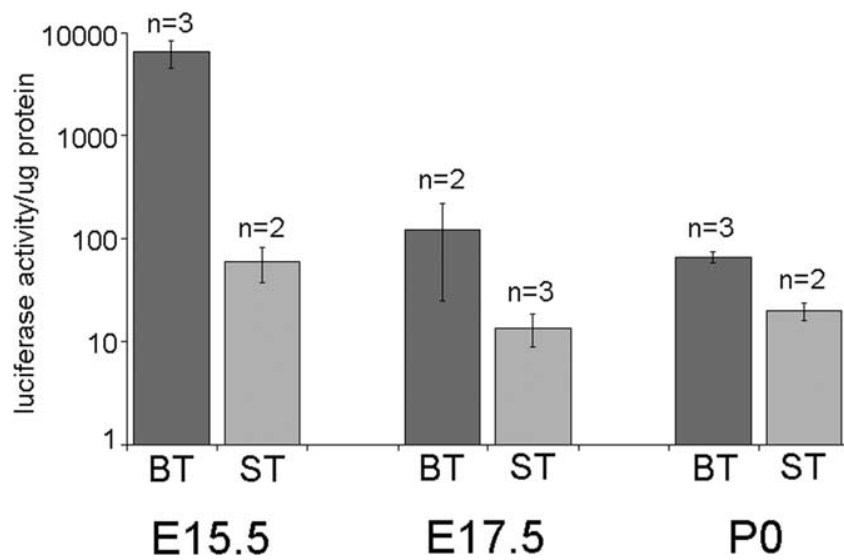

Figure 2. Reduction in transgene expression after Dox withdrawal. The CAErbB2 transgene has an internal ribosomal entry sequence and a firefly luciferase open reading frame cloned in-frame downstream. Thus, from one mRNA, two proteins, CAErbB2 and luciferase, are translated. Hence, luciferase activity was used as surrogate for CAErbB2 expression. Diaphragm muscle extracts were used to measure luciferase activity. At E15.5, when Dox-food was removed, average luciferase activity is high in extracts from BT diaphragm. By E17.5, average luciferase activity is reduced nearly 100 -fold. By birth, average luciferase activity is reduced to levels in ST controls. Results are presented as mean \pm SD.

(see also Fig. 6B). Additional characterization of the restored synapses in $\mathrm{BT}$ muscle at $\mathrm{P} 0$ showed that the area covered by the AChRs was similar to control synapses $(n=6$ for each ST and BT; $p=0.9051$, Student's $t$ test). In addition, terminal Schwann cells as well as acetylcholinesterase and MuSK were present at BT synapses (supplemental Fig. 1, available at www.jneurosci.org as supplemental material). This latter result suggests that AChR cluster formation in BT muscle is also MuSK dependent. Thus, synapses are restored in BT diaphragm by birth after withdrawal of Dox. They appear similar to control synapses except that they localize over the entire surface of the muscle.

\section{$A C h R$ transcription reflects spatial distribution of restored synapses}

We next checked the distribution of $A C h R$ transcripts in our animals. In situ labeling of $A C h R \alpha$ mRNA was done after activation of CAErbB2 until E15.5. The AChR $\alpha$ mRNA in ST animals was highly concentrated in the central zone of muscle fibers as expected (Fig. 5A). Conversely, high levels of $A C h R \alpha$ mRNA were detected throughout the entire length of the muscle fibers in BT diaphragms at E15.5 (Fig. 5B). These results are consistent with our previous analysis of $A C h R \alpha$ mRNA in E.18.5 BT embryos and with the observed threefold increase in $\mathrm{AChR} \alpha$ protein levels in the same muscles (Ponomareva et al., 2006). High levels of transcript could result from the combination of synaptic contact loss (i.e., denervation) and the activity of CAErbB2 in directly stimulating $A C h R \alpha$ transcription throughout the muscle fiber. We also performed in situ hybridization for $A C h R \alpha$ and $A C h R \varepsilon$ mRNAs at $\mathrm{P} 0$ in animals that were given Dox-containing rodent food from E11.5 until E15.5. Whereas control littermates showed the expected central localization of receptor transcript (Fig. $5 C, E)$, labeling for $A C h R \alpha$ and $A C h R \varepsilon$ mRNA in experimental animals showed that there were discreet focal accumulations of them throughout the muscle, which were present in a pattern resembling the distributed AChR cluster staining seen before (Fig. 5D,F). The signal for $A C h R \varepsilon$ transcript was rather weak here in both ST and BT muscle, because its normal peak of expression occurs at approximately P7. Thus, after the removal of Dox, $A C h R$ mRNA localization changes from a diffused to a focal distribution in BT muscle. This change reflects the process of synapse formation and maturation that occurs after Dox withdrawal. The presence of $A C h R \varepsilon$ mRNA in the restored synapses, which is normally seen only after nerve contact, further confirms the presence of the nerve at these sites.

\section{Aneural AChR clusters are observed shortly after Dox withdrawal}

So far, the results seem to support the nerve-dependent model because synapses appeared to form without a preexisting AChR clustering pattern (actually AChR clusters were absent at the time of Dox withdrawal) (Fig. 1), or $A C h R$ transcription pattern; in fact, there were high levels of $A C h R$ transcript throughout the entire muscle at E.15.5 (Fig. 5). Nevertheless, because we cannot observe individual synaptic sites over time, we could not rule at this time that aneural AChR clusters formed after Dox withdrawal and that these would serve as initiation sites for synaptogenesis. To address this possibility, we first examined whether there were aneural AChR clusters ahead of extending nerves during the period of recovery. Whereas immunostaining of recovery animals showed that all distributed AChR clusters at P0 colocalized with nerve, we stained muscle from E16.5 and E17.5 embryos to look for clusters ahead of extending axons. Clusters were examined in three to five fields of diaphragm muscle (see Materials and Methods). These fields were localized in areas distal to the more distant nerve branches. Because many nerve branches were already close to the tendons by E16.5 in BT diaphragm (Fig. 6A), the scored fields in these muscles were near the tendons. For ST muscles, conversely, the scored fields were closer to the center of the muscle. Clusters that did not colocalize with nerve and are described here as aneural were tallied (see the example in Fig. $6 A$ ). In ST diaphragm, only $\sim 2 \%$ of clusters were aneural at E.16.5, whereas $\sim 33 \%$ of AChR clusters in BT diaphragm were aneural (Fig. 6B). In BT diaphragm, only $10 \%$ of clusters were found to be aneural at E17.5. By birth, no aneural AChR clusters were observed and all clusters were colocalized with a nerve terminal in both ST and BT muscle (Fig. 6B). If the data are expressed as average of clusters per diaphragm (supplemental Fig. 2, available at www.jneurosci.org as supplemental material), statistical analysis shows a significant difference in aneural AChR clusters at E16.5 between BT and ST muscles ( $p=0.03$, twotailed $t$ test). No statistical difference was found in nerveassociated clusters between BT and ST muscles at E16.5 (supplemental Fig. 2, available at www.jneurosci.org as supplemental material). Thus, a significant fraction of aneural AChR clusters were found shortly after Dox withdrawal. Their numbers rapidly decline, suggesting that processes leading to synapse formation and maturation are at work during this time.

\section{Nerve is not necessary to generate diffused pattern of AChR clusters}

The mere presence of aneural AChR clusters does not rule out the possibility that synaptogenesis after Dox withdrawal in BT mice is induced by the nerve. For example, some of these aneural AChR clusters could result from the release of agrin from growth cones. To test for the necessity of the nerve to induce the spatial pattern of synapses observed in BT diaphragm after removal of Dox, we introduced into our BT animals a mutation in the HB9 transcription factor by crossing with $\mathrm{Hb}^{+/-}$mice. HB9 null animals lack innervation in diaphragm muscles attributable to phrenic nerve misrouting (Arber et al., 1999; Thaler et al., 1999; Yang et al., 2001). We thus generated $r t \mathrm{TA}^{+/-} ; \mathrm{CAErbB2}^{+/-} ; \mathrm{Hb}^{-/-}$(BT $\mathrm{Hb}^{-1-}$ ) embryos and used as controls $\mathrm{rtTA}^{+/-}$; $\mathrm{CAErbB2}^{+/-}$; 

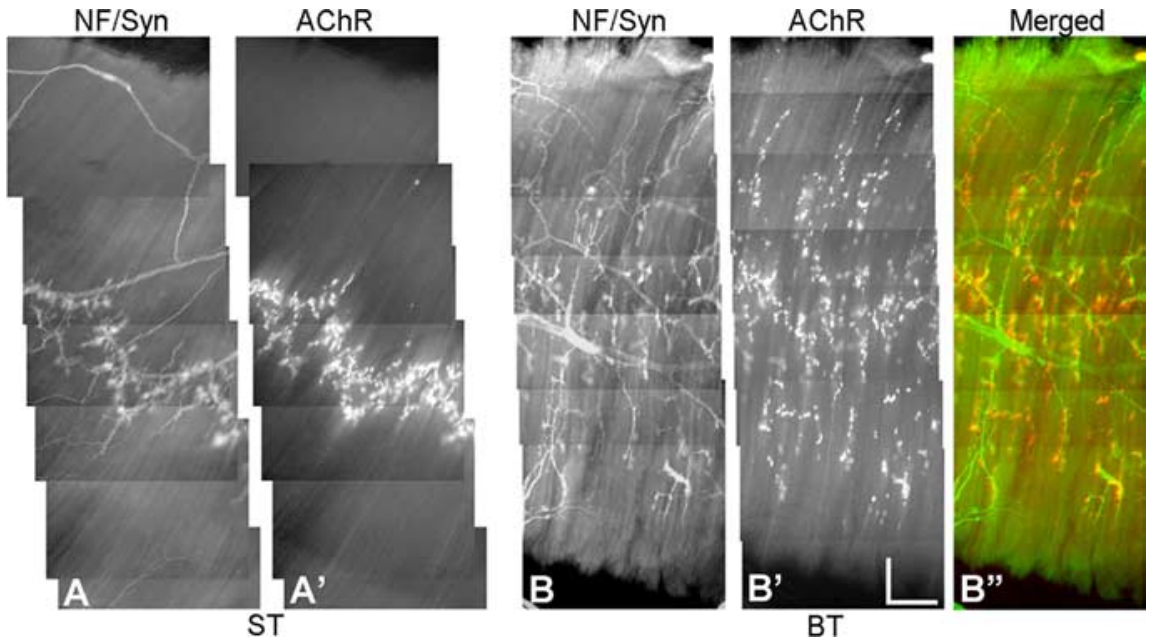

Figure 3. Widely distributed pattern of innervation in BT animals after transient CAErbB2 induction. Whole mounts of diaphragm muscle from POBT and ST animals were stained for $A C h R s$ and axons/nerve terminals as in Figure 1. Images were aligned in a montage to show the pattern of innervation from one side of diaphragm to the other. $A, A^{\prime}$, ST muscle shows central, focal patterning of both nerve and nerve terminal $(\boldsymbol{A})$ with colocalized $A C h R$ clusters $\left(\boldsymbol{A}^{\prime}\right) . \boldsymbol{B}-\boldsymbol{B}^{\prime \prime}, \mathrm{BT}$ animals showed extensive nerve outgrowth $(\boldsymbol{B})$ and $A C h R$ clusters that were distributed throughout the width of diaphragm $\left(\boldsymbol{B}^{\prime}\right)$. A color overlay shows the colocalization of nerve terminal and receptor clusters $\left(\boldsymbol{B}^{\prime \prime}\right)$. Scale bars, $200 \mu \mathrm{m}$.
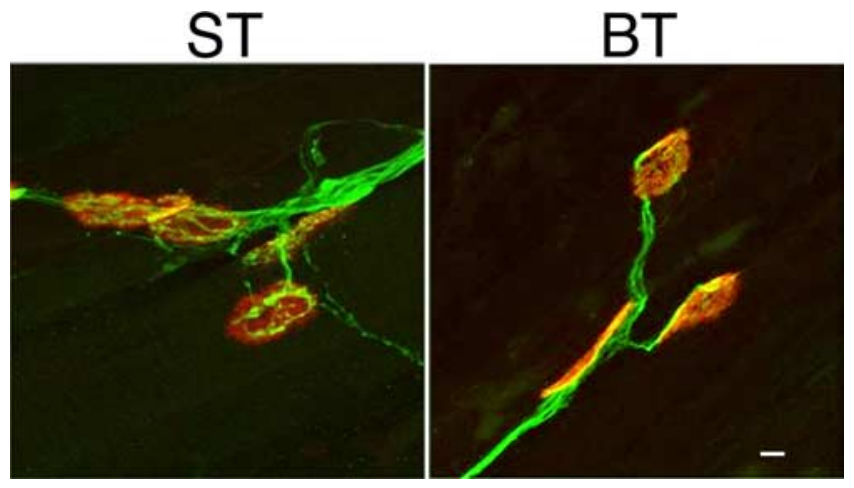

Figure 4. Proper presynaptic and postsynaptic apposition in restored synapses after transient CAErbB2 induction. Representative high-power, maximal confocal projections of synapses in ST and BT P0 diaphragms after transient embryonic CAErbB2 induction. Axon/nerve termina markers in green, and AChRs in red. No aneural AChR clusters were observed at this time. Images such as these were used to estimate AChR cluster area (see Results). Scale bar, $5 \mu \mathrm{m}$.

$\mathrm{Hb9}^{+/ ?}\left(\mathrm{BT} \mathrm{Hb}^{+/ ?}\right)$ or $\mathrm{rtTA}^{-/-}$; $\mathrm{CAErbB2} 2^{+/-} ; \mathrm{Hb}^{+/ ?}$ (ST $\mathrm{Hb}^{+/ ?}$ ) littermates. We fed Dox-containing food to pregnant dams from E11.5 until E15.5. Whole-mount immunostaining of embryonic diaphragm muscle was done to determine whether receptor clusters were present in E15.5 and E18.5 tissue and, if found, where they would localize. As observed in BT mice (Fig. 1), no AChR clusters were detected at E15.5 in BT $\mathrm{Hb}^{-/-}$mice (supplemental Fig. 3, available at www.jneurosci.org as supplemental material). Figure 7 shows collages of entire sections of diaphragms from embryos of different genotypes at E18.5. As observed at P0, BT $\mathrm{Hb}^{+/ \text {? }}$ diaphragm at E18.5 showed a diffused pattern of synapses across the entire muscle, whereas ST $\mathrm{Hb}^{+/ \text {? }}$ controls showed the typical concentration of synapses in the center on the muscle (Fig. $7 A-B^{\prime}$ ). Diaphragms from BT $\mathrm{Hb}^{-1-}$ embryos, which lacked phrenic nerve (Fig. $7 C^{\prime}$ ), showed a distribution of aneural AChR clusters (Fig. 7C) very similar to the diffused distribution of synapses in $\mathrm{BT} \mathrm{Hb}^{+/ \text {? }}$ diaphragms, which had innervation by phrenic nerve. The distribution of aneural AChR clusters in BT $\mathrm{Hb}^{-/-}$diaphragms from animals that were given Dox from E11.5 to E15.5 (Fig. 7C) was also very difE18.5 BT $\mathrm{Hb}^{-/-}$diaphragm for AChR and MuSK. We found that aneural AChR clusters in these muscles costained for MuSK (supplemental Fig. 4, available at www.jneurosci.org as supplemental material). Thus, the formation of aneural AChR clusters in BT muscle is also MuSK dependent.

\section{Discussion}

By transient expression of CAErbB2 selectively in developing muscle, we forced the nerve and muscle to engage in synaptogenesis without a central prepattern of AChR clusters or AChR transcription. A widely diffused distribution of synapses throughout the muscle resulted. Under the nerve-dependent model of synaptogenesis, this distribution would have been explained by the wide pattern of axonal sprouting induced by CAErbB2 expression. Yet, in the absence of the nerve, a very similar, muscledependent distribution of aneural AChR clusters was generated. This result is not readily explained by the nerve-dependent model. It, together with the significant proportion of aneural AChR clusters observed shortly after Dox withdrawal, suggest that the muscle may be setting the site for initiation of synaptogenesis in our experimental paradigm. It could be argued that the aneural AChR clusters observed in BT $\mathrm{Hb}^{-/-}$muscle are just spontaneously formed "hotspots," whose significance for nerveinduced synapse formation is minimal. Because live imaging of mammalian neuromuscular synaptogenesis is not feasible at present, we cannot completely rule the possibility the nerve induces clusters de novo at sites in which synapses form. However, the fact that a similar pattern of clusters forms in both the presence and absence of the nerve suggests that this is not the case. Moreover, vital imaging in zebrafish has shown that preexisting AChR clusters are the preferential sites for synapse formation (Flanagan-Steet et al., 2005; Panzer et al., 2006).

What results would have supported the nerve-dependent model? Because AChR clusters were absent by E15.5, one possibility was that no AChR clusters would have reappeared by E18.5 in BT $\mathrm{Hb}^{-1-}$ mice. That is, all new AChR clusters would be nerve dependent. This was clearly not the case because many aneural AChR clusters formed in BT $\mathrm{Hb}^{-/-}$embryos. Another possibility that would have supported the nerve-dependent 
ST
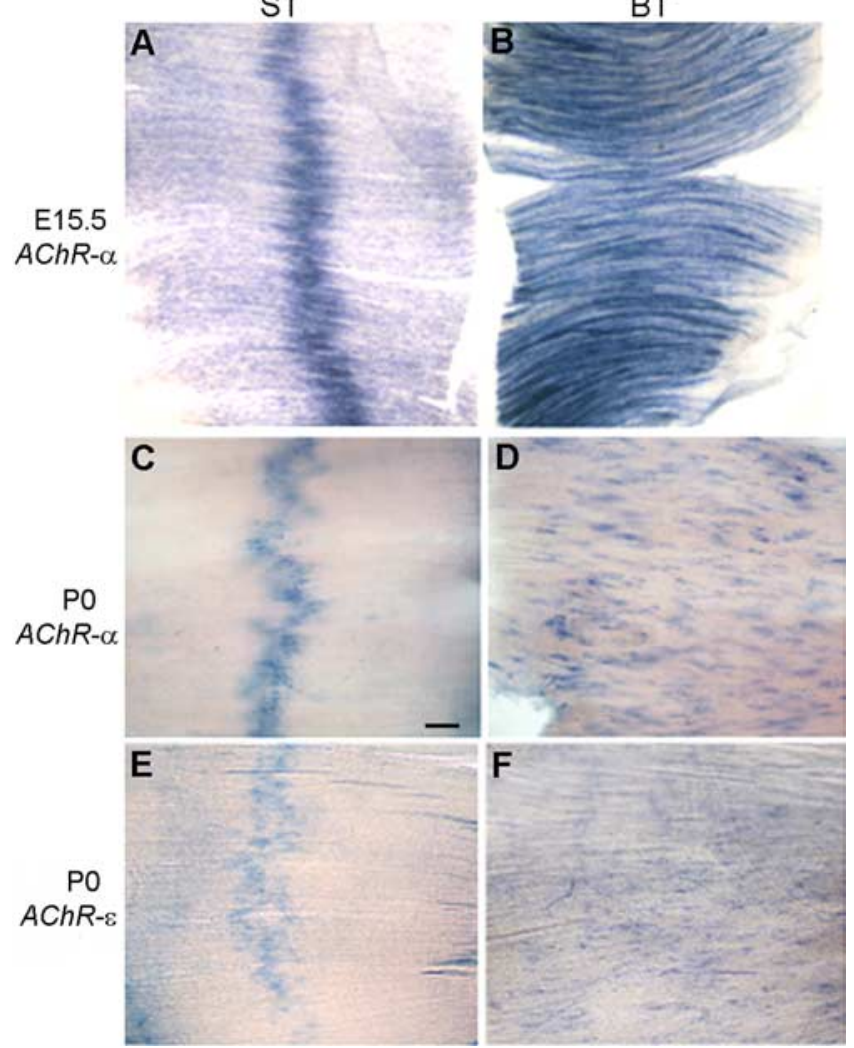

Figure 5. Changes in pattern of $A C h R$ transcription after transient CAErbB2 induction. $\boldsymbol{A}$ Nonradioactive whole-mount in situ hybridization for AChR $\alpha$ mRNA in a control ST E15.5 diaphragm shows transcript concentration in a narrow band in the middle of the muscle. $\boldsymbol{B}, \ln B T$ diaphragm, high levels of expression occur throughout the length of muscle fibers. $C$, P0 diaphragm from ST animal was labeled for AChR $\alpha$ mRNA and shows a tight band and central localization for $A C h R \alpha$ transcription. $D$, In BT animals labeled for $A C h R \alpha$ mRNA, the distinct focal concentrations of transcription occur throughout the width of diaphragm. This appears to mirror the distribution of synapses revealed by protein staining (Fig. 3). E, AChR $\varepsilon$ mRNA, which is only found localized at synaptic sites, was labeled. In ST animals, it is present in a centrally concentrated pattern. $\boldsymbol{F}$, In BT animals, AChR $\varepsilon$ mRNA appears distributed throughout the diaphragm in distinct focal concentrations. Scale bar, $100 \mu \mathrm{m}$.

model was that aneural AChR clusters that reformed in BT $\mathrm{Hb}^{-/-}$diaphragm were restricted to the central area of the muscle. That is, outside the central zone, only nerve-induced synaptogenesis would occur as a result of axonal sprouting. This was also not case because the distribution of aneural AChR clusters in BT $\mathrm{Hb}^{-/-}$diaphragms from embryos that received Dox (Fig. 7C) was much wider than that in diaphragms from BT $\mathrm{Hb}^{-1-}$ embryos that received no Dox (Fig. 7D). This result also suggests that transient, skeletal muscle-selective CAErbB2 expression leads to the widening of the aneural AChR prepattern.

What is the molecular mechanism(s) underlying the centralto-diffused distribution of NMJs after transient CAErbB2 expression? Although the precise mechanism(s) involved remains unknown, we suggest the following.

(1) As shown in Figure 5, CAErbB2 expression led to relatively uniform, high $A C h R$ mRNA expression throughout the muscle fibers by E15.5. This suggests that, by expressing CAErbB2, we simply widened the transcriptional prepattern of $A C h R$, and presumably other postsynaptic genes, from the central zone to the entire muscle fiber length. After Dox was withdrawn and CAErbB2 levels went below an unknown threshold, the nerve and muscle engaged in synaptogenesis, apparently following the rules during normal development except that they had a much wider
A

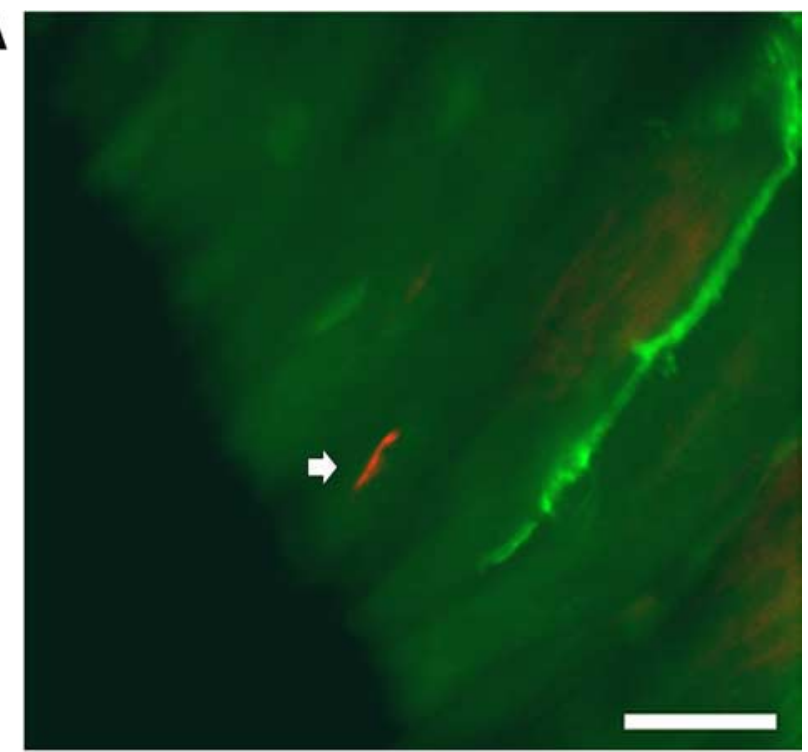

B

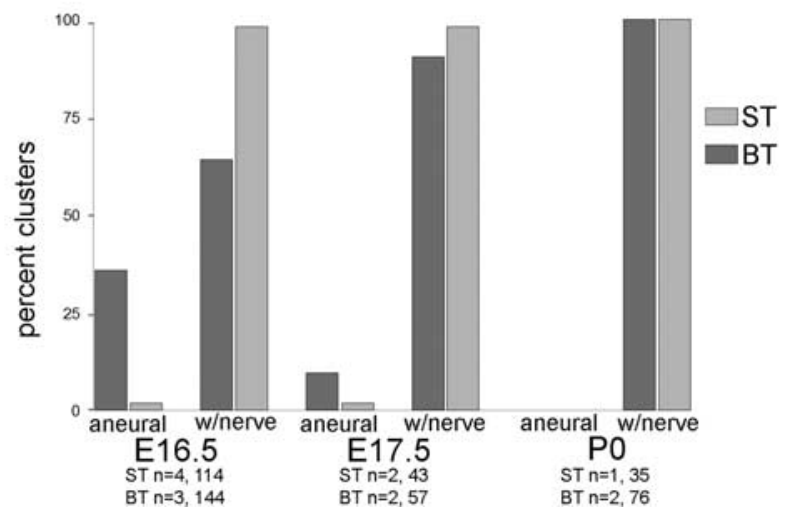

Figure 6. Aneural AChR clusters are detected early after Dox withdrawal but disappear by birth. Diaphragm muscle from E16.5 and E17.5 embryos and P0 neonates was labeled for axons, nerve terminals, and AChRs as above. $A$, An example of an aneural AChR cluster (arrow) seen near the edge of a E16.5 diaphragm muscle. Scale bar, $25 \mu \mathrm{m}$. $\boldsymbol{B}$, Histogram showing the percentage of clusters counted that were colocalized with nerve terminal, and the percentage that were deemed aneural or where no nerve staining was detected at the site of AChR cluster. AtE16.5, ST embryos had 2\% aneural clusters, whereas in BT embryos, approximately one-third of total clusters counted were aneural. At E17.5, ST embryos showed nearly no aneural clusters, whereas in BT animals, 1/10 of total clusters were aneural. By birth, however, in both ST and BT animals, no aneural clusters were detected; $n=$ number of diaphragms, total number of clusters.

field to do so, set by the width of the transcriptional pattern. To us, this seems the simpler and more likely mechanism. This interpretation raises the possibility that what sets the location and pattern of mammalian neuromuscular innervation is the width and location of the postsynaptic transcriptional pattern. Consistent with this conclusion, sensory and motor neuron conditional neuregulin-1 mutant mice display exuberant axonal sprouting of the phrenic nerve yet show a central narrow band of AChR clustering and $A C h R$ transcription in their diaphragm (Yang et al., 2001). One NMJ per muscle fiber is the norm for most mammalian muscles. However, there are examples of multipleinnervated muscles such as the extraocular and laryngeal muscles (cf. Khanna et al., 2003). Although technically challenging, it would be interesting to examine the AChR clustering and transcriptional patterns in those muscles during development. Perhaps, multiple "prepatterns" would also be observed. (2) CAErbB2 expression could also both stimulate the production of 


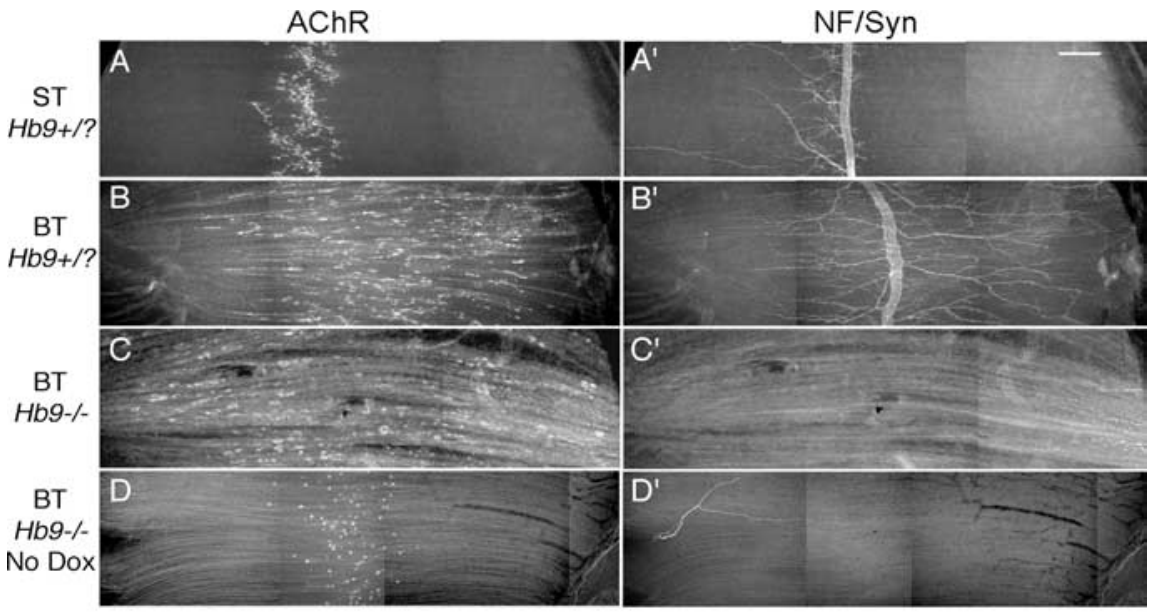

E
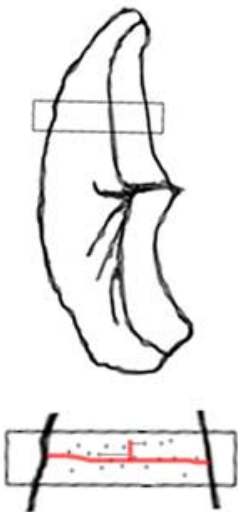

F Avg. cluster distance from midpoint

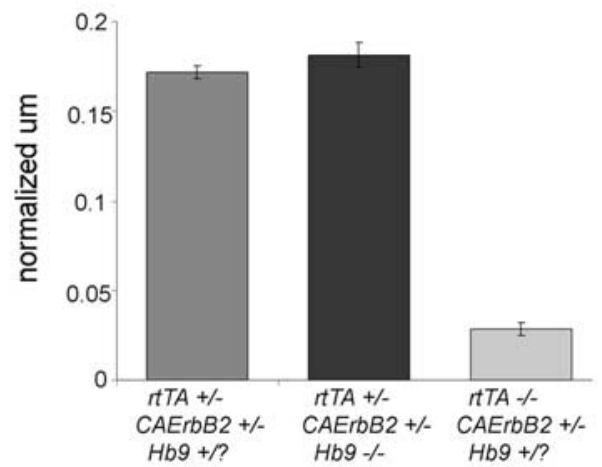

Figure 7. Distributed pattern of $A C h R$ clustering is nerve independent. $A-D^{\prime}$, Whole mounts of diaphragm muscle from E18.5 embryos were labeled for axons/nerve terminals and AChRs as above. Epifluorescence microscopy was used to collect images that were aligned into montages to show the distribution of AChR clustering from end to end of diaphragms. $A$, ST animals displayed central pattern of AChR clustering and corresponding colocalization of central nerve bundle and nerve terminals $\left(\boldsymbol{A}^{\prime}\right)$. Scale bar, $50 \mu \mathrm{m} . \boldsymbol{B}, \mathrm{BT}$ animals had cluster distribution that was demonstrated previously (Fig. 3), in which clusters were located throughout the width of diaphragm muscle and in which neuronal outgrowth resulted in nerve terminals that spread throughout diaphragm muscle $\left(\boldsymbol{B}^{\prime}\right)$. C, When BT animals were also $\mathrm{Hb} 9$ null, AChR clusters were also found throughout the width of diaphragm muscle. $\boldsymbol{C}^{\prime}$, Motor axons were absent from muscle as expected. $\boldsymbol{D}$, Diaphragm muscle from BT $H b 9^{-\prime-}$ embryo that was not induced with Dox. AChR clusters appear in a centrally localized band, which is slightly wider than in ST controls but much narrower than in $\mathrm{BT} H b 9^{-/-}$embryos that received Dox. $D^{\prime}$, Nerve staining shows absence of motor axons while a sensory nerve is visible. E, Diagram of hemidiaphragm showing region of muscle used for images and determination of AChR clusters distribution. An enlarged diagram below shows how cluster distribution was measured. Width of diaphragm was determined, and then a fraction of that width was used to determine the height of measurement region. Distances of 20 individual clusters from the midpoint of each diaphragm were collected. $\boldsymbol{F}$, Histogram showing average cluster distance comparisons between several genotypes. For each genotype, 20 cluster distances were measured from each animal, making a total of 60 clusters for each of the $\mathrm{BT}$ genotypes and 40 clusters for the ST embryos ( $n=3$ and $n=2$, respectively).

muscle-derived axonal sprouting factors and/or inhibit the synthesis of adhesion molecules that normally stop the axon from growing beyond the central zone. This would only account for the exuberant axonal growth, not for the onset of AChR clustering, because the experiment with the BT $\mathrm{Hb}^{-1-}$ mice showed that the muscle itself can account for the latter. In the presence of the nerve, the combined activity of ACh and agrin would ensure the proper maturation of the aneural AChR sites into synaptic sites. (3) It is possible that, by expressing CAErbB2, we altered the normal timing and expression of the endogenous muscle differentiation program. In this context, mice null for the myogenic transcription factor MyoD display a diffused pattern of neuromuscular innervation that is evident beginning at E15.5 and persists into the adult (Wang et al., 2003). However, unlike our CAErbB2-expressing mice, in $\mathrm{MyoD}^{-1-}$ mice, AChR clustering per se was not abolished at any time. The spatial pattern of $A C h R$ transcription was not studied in these mice.

Regardless of the underlying mecha$\operatorname{nism}(\mathrm{s})$, transient, skeletal muscle-specific expression of CAErbB2 leads to reprogramming of the endogenous muscle AChR prepattern. In turn, this reprogramming, and not the nerve, seems responsible for the widely distributed pattern of synapses in BT neonates. However, the possibility that the prepattern reprogramming induced by CAErbB2 results from changes in muscle development calls for caution in the interpretation of the present results.

In conclusion, we sought to provide a test in mice for the two models of neuromuscular synaptogenesis that have been proposed from previous studies in mice and zebrafish. Our results appear to support the muscle-dependent, prepattern model because the presence of the nerve seems unnecessary to generate the widely diffused innervation pattern we observed in mice transiently expressing CAErbB2.

\section{References}

Arber S, Han B, Mendelsohn M, Smith M, Jessell TM, Sockanathan S (1999) Requirement for the homeobox gene Hb9 in the consolidation of motor neuron identity. Neuron 23:659-674.

Arber S, Burden SJ, Harris AJ (2002) Patterning of skeletal muscle. Curr Opin Neurobiol 12:100-103.

Braissant O, Wahli W (1998) A simplified in situ hybridization protocol using non-radioactively labeled probes to detect abundant and rare $\mathrm{mR}$ NAS on tissue sections. Biochemica 1:10-16.

Flanagan-Steet H, Fox MA, Meyer D, Sanes JR (2005) Neuromuscular synapses can form in vivo by incorporation of initially aneural postsynaptic specializations. Development 132:4471-4481.

Khanna S, Richmonds CR, Kaminski HJ, Porter JD (2003) Molecular organization of the extraocular muscle neuromuscular junction: partial conservation of and divergence from the skeletal muscle prototype. Invest Ophthalmol Vis Sci 44:1918-1926.

Kummer TT, Misgeld T, Sanes JR (2006) Assembly of the postsynaptic membrane at the neuromuscular junction: paradigm lost. Curr Opin

Neurobiol 16:74-82.

Lin W, Burgess RW, Dominguez B, Pfaff SL, Sanes JR, Lee KF (2001) Distinct roles of nerve and muscle in postsynaptic differentiation of the neuromuscular synapse. Nature 410:1057-1064.

Lin W, Dominguez B, Yang J, Aryal P, Brandon EP, Gage FH, Lee KF (2005) Neurotransmitter acetylcholine negatively regulates neuromuscular synapse formation by a Cdk5-dependent mechanism. Neuron 46:569-579.

McMahan UJ (1990) The agrin hypothesis. Cold Spring Harb Symp Quant Biol 55:407-418.

Misgeld T, Kummer TT, Lichtman JW, Sanes JR (2005) Agrin promotes synaptic differentiation by counteracting an inhibitory effect of neurotransmitter. Proc Natl Acad Sci USA 102:11088-11093.

Panzer JA, Gibbs SM, Dosch R, Wagner D, Mullins MC, Granato M, BaliceGordon RJ (2005) Neuromuscular synaptogenesis in wild-type and mutant zebrafish. Dev Biol 285:340-357.

Panzer JA, Song Y, Balice-Gordon RJ (2006) In vivo imaging of preferential motor axon outgrowth to and synaptogenesis at prepatterned acetylcho- 
line receptor clusters in embryonic zebrafish skeletal muscle. J Neurosci 26:934-947.

Ponomareva ON, Ma H, Vock VM, Ellerton EL, Moody SE, Dakour R, Chodosh LA, Rimer M (2006) Defective neuromuscular synaptogenesis in mice expressing constitutively active ErbB2 in skeletal muscle fibers. Mol Cell Neurosci 31:334-345.

Rimer M, Prieto AL, Weber JL, Colasante C, Ponomareva O, Fromm L, Schwab MH, Lai C, Burden SJ (2004) Neuregulin-2 is synthesized by motor neurons and terminal Schwann cells and activates acetylcholine receptor transcription in muscle cells expressing ErbB4. Mol Cell Neurosci 26:271-281.

Schaeren-Wiemers N, Gerfin-Moser A (1993) A single protocol to detect transcripts of various types and expression levels in neural tissue and cultured cells: in situ hybridization using digoxigenin-labelled cRNA probes. Histochemistry 100:431-440.

Thaler J, Harrison K, Sharma K, Lettieri K, Kehrl J, Pfaff SL (1999) Active suppression of interneuron programs within developing motor neurons revealed by analysis of homeodomain factor HB9. Neuron 23:675-687.

Wang ZZ, Washabaugh CH, Yao Y, Wang JM, Zhang L, Ontell MP, Watkins SC, Rudnicki MA, Ontell M (2003) Aberrant development of motor axons and neuromuscular synapses in MyoD-null mice. J Neurosci 23:5161-5169.

Yang X, Li W, Prescott ED, Burden SJ, Wang JC (2000) DNA topoisomerase IIbeta and neural development. Science 287:131-134.

Yang X, Arber S, William C, Li L, Tanabe Y, Jessell TM, Birchmeier C, Burden SJ (2001) Patterning of muscle acetylcholine receptor gene expression in the absence of motor innervation. Neuron 30:399-410.

Zuo Y, Lubischer JL, Kang H, Tian L, Mikesh M, Marks A, Scofield VL, Maika S, Newman C, Krieg P, Thompson WJ (2004) Fluorescent proteins expressed in mouse transgenic lines mark subsets of glia, neurons, macrophages, and dendritic cells for vital examination. J Neurosci 24:1099911009 . 